\title{
Les Fab Labs et " ateliers numériques » en France
}

\section{François Piuzzi (piuzzifr@gmail.com)}

Président de la Commission «Physique sans Frontières »

des Sociétés Françaises de Physique et d’Optique

Les Fab Labs ou Laboratoires

\section{de Fabrication constituent}

un nouveau type de lieu où,

\section{à l'aide principalement}

de machines numériques,

\section{s'effectuent la conception}

\section{et la réalisation d'objets}

et de prototypes. Ce sont

également des sites d'échanges,

de collaboration et de formation.

Plutôt destinés à leur origine

à des citoyens attirés par leurs

possibilités, ils se sont développés

récemment dans les mondes

académique et industriel.

Leur apport pour les temps

de pandémie, ainsi que

leur importance pour l'Afrique,

sont discutés ici.

\section{Qu'est-ce qu'un Fab Lab ?}

En bref, un Fab Lab est un Laboratoire de Fabrication, un site d'échanges en accès libre autour de la réalisation d'objets plus ou moins complexes type robots, à l'aide principalement de machines numériques, mais aussi grâce à des moyens plus conventionnels d'établis pour des opérations de finition ou d'assemblage, et avec une méthodologie particulière liée au partage des expériences et des informations. La définition et les règles du fonctionnement des Fab Labs sont explicitées dans une charte [1] mise en place par le MIT. Seules les structures respectant cette charte peuvent afficher le logo officiel (fig. 1). À leurs débuts, les Fab Labs étaient proches du paradigme du DIY "Do It Yourself", ou (à la française) du "Système D ». Un très bon article leur est consacré sur Wikipédia.

Un Fab Lab met à disposition de l'utilisateur (après une formation) une panoplie de machines numériques, une découpeuse laser, une fraiseuse, une imprimante $3 \mathrm{D}$, l'incontournable fer à souder, éventuelle-

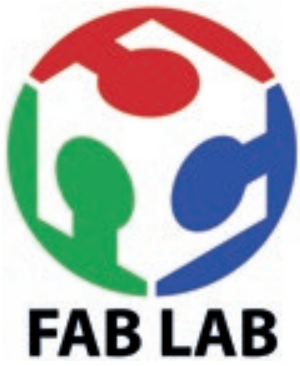

1. Le logo des Fab Labs.

ment un scanner 3D pour la reproduction d'objets, ainsi que des outils de modélisation numérique. Pour certains sites, il y a un accès à la formation et au développement de logiciels pour les microcontrôleurs de la plateforme Arduino ${ }^{(a)}$ et à la programmation sous Python des nano-ordinateurs Raspberry Pi $\mathrm{i}^{(\mathrm{b})}$. Des formations à l'utilisation des logiciels de création d'objets en 3D comme OpenSCAD (on ne devient pas concepteur 3D d'un coup !) et des logiciels de pilotage des différentes machines sont également assurées.

De nombreux matériaux peuvent être utilisés : bois, métaux, plastiques, tissus, etc. Les objets fabriqués sont très divers, allant

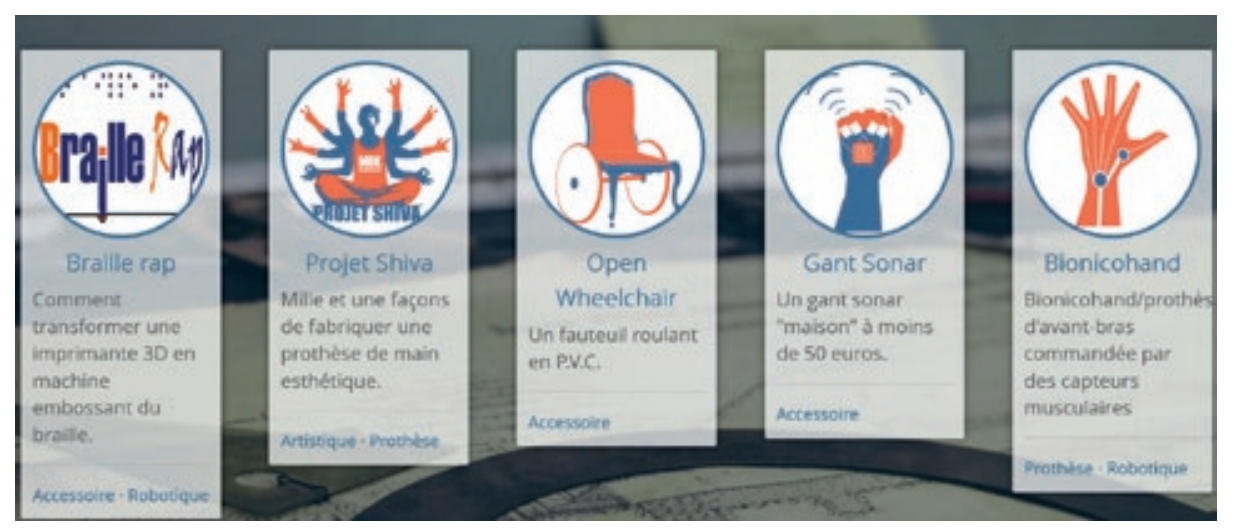

2. Cinq projets d'aide technique aux handicapé.e.s, réalisés dans le Fab Lab de l'association My Human Kit. (https://myhumankit.org) 


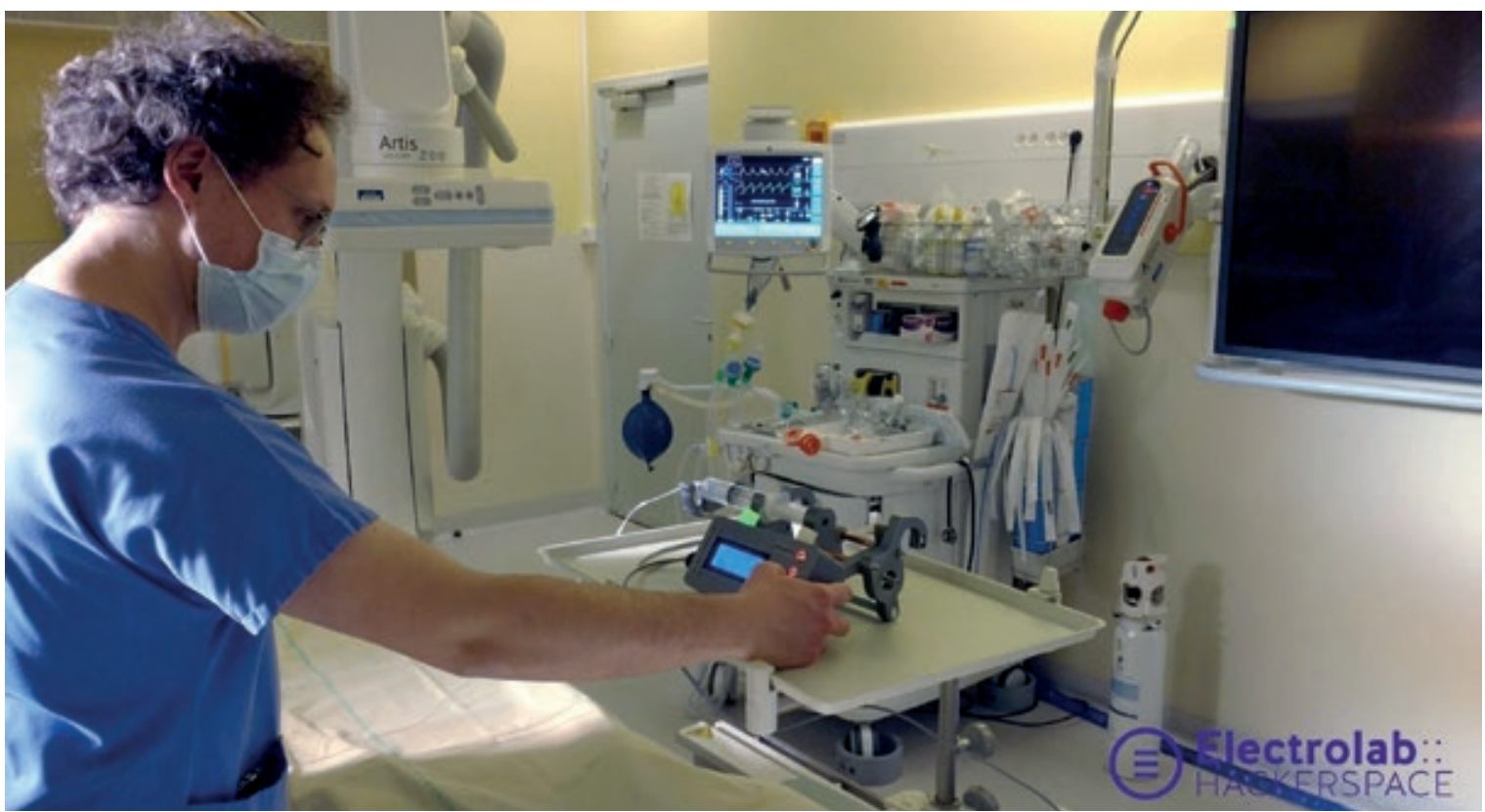

Essai de prise en main en conditions réelles à l'Hôpital Lariboisière à Paris, le 12 avril 2020, du pousse-seringue électrique open source développé par le hackerspace Electrolab à Nanterre (voir p. 35 et fig. 5).

de la toupie à l'objet intelligent (quelquefois connecté) en passant par le robot ou la main articulée pour prothèse. Un grand avantage est la fabrication de pièces impossibles à réaliser par moulage. Dès qu'un projet est terminé, il est demandé qu'une documentation soit établie pour que le projet puisse être publié et donc partagé sur Internet, en général en utilisant la plateforme GitHub. Les activités dont nous ne parlerons pas dans cet article sont celles concernant la couture, la décoration et la cuisine, qui cependant donnent lieu à des développements intéressants comme la fabrication de masques par des couturier.es à l'époque du Covid-19.

Les avantages de ces sites de fabrication d'innovations sont multiples : les nombreuses compétences qui y sont regroupées en font des endroits multi et transdisciplinaires. Les Fab Labs sont ouverts à tous (pas d'élitisme). Ils se basent sur une communauté où les utilisateurs prennent part à la capitalisation des connaissances, à la formation et aux échanges avec les autres utilisateurs, notamment autour de compétences techniques ou logicielles, et à l'émergence d'idées nouvelles. Le lancement de concours sur des thèmes précis permet souvent d'arriver à des solutions originales et pour des couts très bas. Il faut remarquer que cette approche participative induit une augmentation de la créativité. La panoplie de machines dispo- nibles rend possibles des prototypages rapides, ce qui permet la correction des erreurs de conception et l'amélioration rapide du modèle initial. La frugalité est une caractéristique importante des Fab Labs qui, avec le recyclage de composants, contribuent à l'économie circulaire. Enfin, les Fab Labs ont une dimension démocratique, car ils permettent la réalisation de projets qui ne présentent pas (ou que peu) d'intérêt pour une logique marchande, comme des dispositifs d'aide pour les personnes handicapées. Un bel exemple est l'association française My Human Kit [2], dont certains des projets sont présentés dans la figure 2 .

Le succès des Fab Labs a incité des universités, grandes écoles et industries à monter des structures semblables pour les étudiants et les membres du personnel. Leur accès étant restreint, elles ne peuvent pas afficher le logo officiel.

\section{Qui utilise les Fab Labs ?}

Côté utilisateurs : le mouvement a démarré avec beaucoup d'artistes, concepteurs (designers), architectes, anthropologues, des experts en programmation, en informatique, des jeunes "bricoleurs » et " bidouilleurs » de 15 à 90 ans, curieux, avides de fabriquer des objets personnalisés et conçus par euxmêmes, et de trouver avec qui se former et échanger. Il ne faut pas oublier les hackers pour lesquels c'est aussi l'occasion de faire un pied de nez au système, en détournant la technologie et en trouvant des solutions frugales et innovantes à certains problèmes.

Côté permanents : ce sont des passionnés, issus soit du mouvement associatif, soit du personnel universitaire pour le cas des Fab Labs universitaires, soit des étudiants bénévoles, etc.

\section{Pourquoi émergent-ils maintenant (en fait, depuis plusieurs années)?}

Les couts des machines numériques ont beaucoup baissé, en particulier celui des imprimantes 3D. Pour celles-ci, cette baisse est liée au passage dans le domaine public du brevet de "l'impression additive ". De nombreux intervenants se sont emparés d'une technologie désormais mature pour développer des modèles grand public à des prix abordables. Cependant, contrairement à ce qui a été affiché et publicisé, l'utilisation des imprimantes 3D n'est pas encore complètement adaptée au grand public et les temps d'impression sont relativement longs, pouvant dépasser plusieurs heures. Les filaments utilisés pour l'impression sont de plus en plus divers, avec notamment l'inclusion de particules magnétiques ou de nanoparticules de cuivre qui ont un 
$>>$

effet bactéricide (par exemple pour la fabrication de NanoHack, masque imprimé en $3 \mathrm{D}$ et open source pour se protéger contre la Covid-19 [3]), de fibres de différentes natures, et enfin de filaments à base d'algues. Il y a aussi la possibilité de produire des filaments en recyclant le plastique, ce qui présente un intérêt écologique évident et participe à l'économie circulaire.

Les microcontrôleurs de la plateforme Arduino pour l'électronique et les nanoordinateurs Raspberry Pi sont caractérisés par des couts très faibles (quelques dizaines d'euros). Les nano-ordinateurs Raspberry Pi permettent aussi de piloter les imprimantes 3D sans besoin d'un ordinateur dédié. Il y a une convergence naturelle avec le mouvement open source (source ouverte) et les aspects collaboratifs et participatifs.

\section{Imaginer, fabriquer, partager ses idées et savoir-faire, construire une communauté}

La «philosophie » des Fab Labs implique un changement total de paradigme avec la fabrication industrielle classique car, d'une part, on ne fabrique que ce qui est nécessaire et avec le minimum de matière ; d'autre part, il n'y a plus de notion de brevet, les innovations étant partagées sous une "licence " du type Creative Commons ou sous la licence sur le matériel libre du CERN [4]. Cela induit des avantages, puisqu'une innovation peut évoluer grâce à des modifications des plans impulsées par de multiples collaborations. Le résultat est une démocratisation de la conception et de la fabrication. Cependant, cela implique qu'il n'y ait plus de propriété industrielle, ni de logique directe de société marchande. Malheureusement, les limites actuelles de l'utilisation des imprimantes 3D par le " grand public " (voir ci-dessus) et l'impossibilité de la production en grande série qui en résulte, constituent aujourd'hui une limitation.

L'aspect réseau des Fab Labs est aussi une caractéristique importante, car il permet une circulation très rapide des innovations technologiques, plus rapide que dans le système marchand où la survie de l'entreprise est liée au secret de fabrication. Une discussion intéressante sur ce sujet est présentée dans un document publié par des économistes [5].

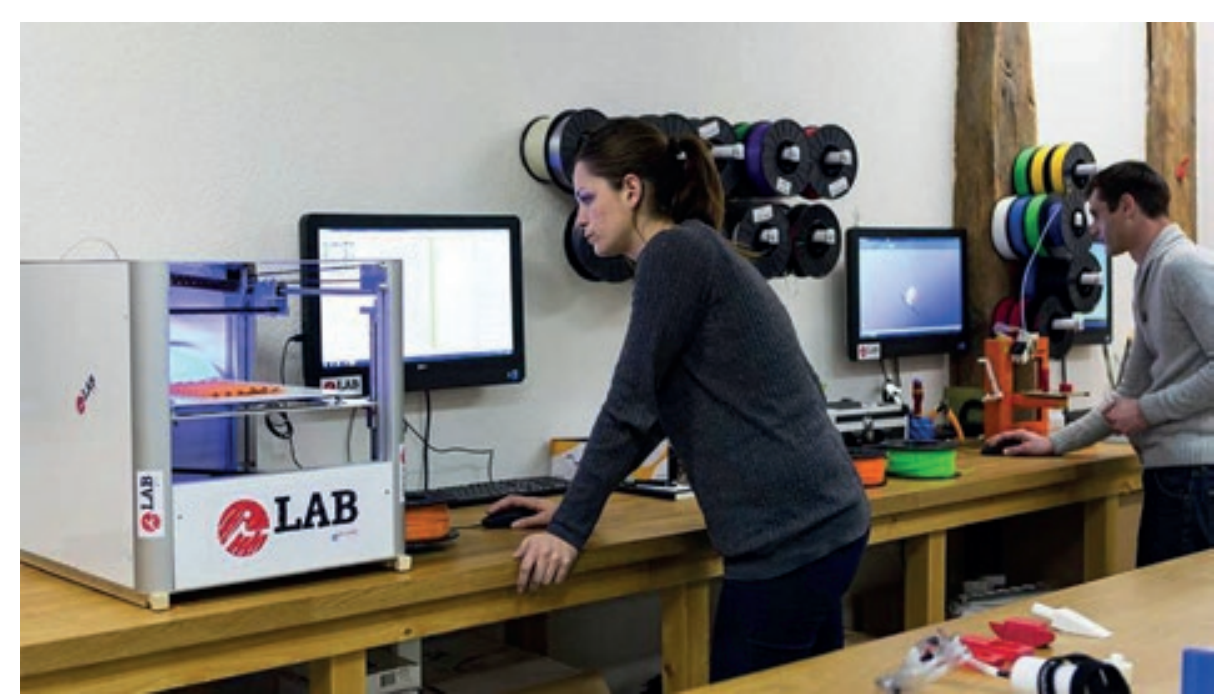

3. Le laboratoire i-Lab d'Air Liquide, à Paris. (Photo www.lesquare.com / Air Liquide).

L'évaluation du nombre actuel de Fab Labs dépend de la seule prise en compte de ceux obéissant à la charte (environ 150 en France et 1750 dans le monde en 2018), ou bien de l'ensemble des ateliers numériques $^{(\mathrm{c})}$. La carte établie par l'association Makery [6] permet d'avoir une idée de la répartition des Fab Labs français, des hackerspaces ${ }^{(\mathrm{d})}$, des " ateliers numériques d'entreprises », etc. Citons quelques exemples intéressants : le Fab Lab de La Casemate à Grenoble, celui de l'université de Cergy Pontoise soutenu par Orange, La Paillasse à Paris, le Photonic FabLab hébergé par le bâtiment 503 à Orsay, et enfin le hackerspace Electrolab à Nanterre (voir plus loin).

On peut trouver des milliers de projets réalisables dans le cadre d'un Fab Lab, dont beaucoup sont caractérisés par une ambition scientifique. Plusieurs sites Internet ${ }^{(\mathrm{e})}$ présentent des dispositifs pour l'éducation scientifique ainsi que pour la vulgarisation. Une revue récente publiée par Elsevier, HardwareX [7], propose des articles sur la conception et la fabrication d'équipements scientifiques à cout raisonnable, dont beaucoup en source ouverte.

\section{Entreprises et Fab Labs}

Dans certaines grandes entreprises la taille peut constituer un handicap pour le domaine de l'innovation, car il est possible que des rigidités structurelles empêchent une collaboration multidisciplinaire efficace. C'est pour cette raison que plusieurs sociétés ont développé en interne des structures de type Fab Lab (c'est le cas de Renault et de Dassault Systèmes, par exemple), car cela permet d'accélérer l'innovation par une augmentation du dialogue, des interactions et des échanges plus efficaces, tout en maintenant la propriété industrielle au sein de l'entreprise. Le cas du i-Lab d'Air Liquide (fig. 3) est également très intéressant.

\section{Fab Labs, ateliers et espaces numériques pour la science citoyenne et le développement}

Le mouvement commence à s'intéresser aux technologies permettant d'arriver à des couts abordables pour, d'une part, développer des instruments en source ouverte (open source) pour la caractérisation des problèmes environnementaux et, d'autre part, pour ce qui concerne l'instrumentation scientifique pour les pays en voie de développement [8]. Dans la première catégorie il y a la structure Public Lab basée à New York, dont le but est de développer des kits d'instruments pour une démocratisation de la science ; dans la deuxième, il y a le Scientific FabLab de l'ICTP à Trieste [9], les « filles » du collectif catalan Gyne Punk qui créent des outils à bas cout pour la gynécologie, et le GaudiLab à Lucerne (Suisse) qui propose toute une série d'instruments de laboratoire pour la biologie, avec l'originalité d'inclure des composants recyclés comme dans leur remarquable dispositif de pinces optiques. Le dernier développement du GaudiLab est une plateforme miniature pour la PCR («manière d'obtenir plusieurs 


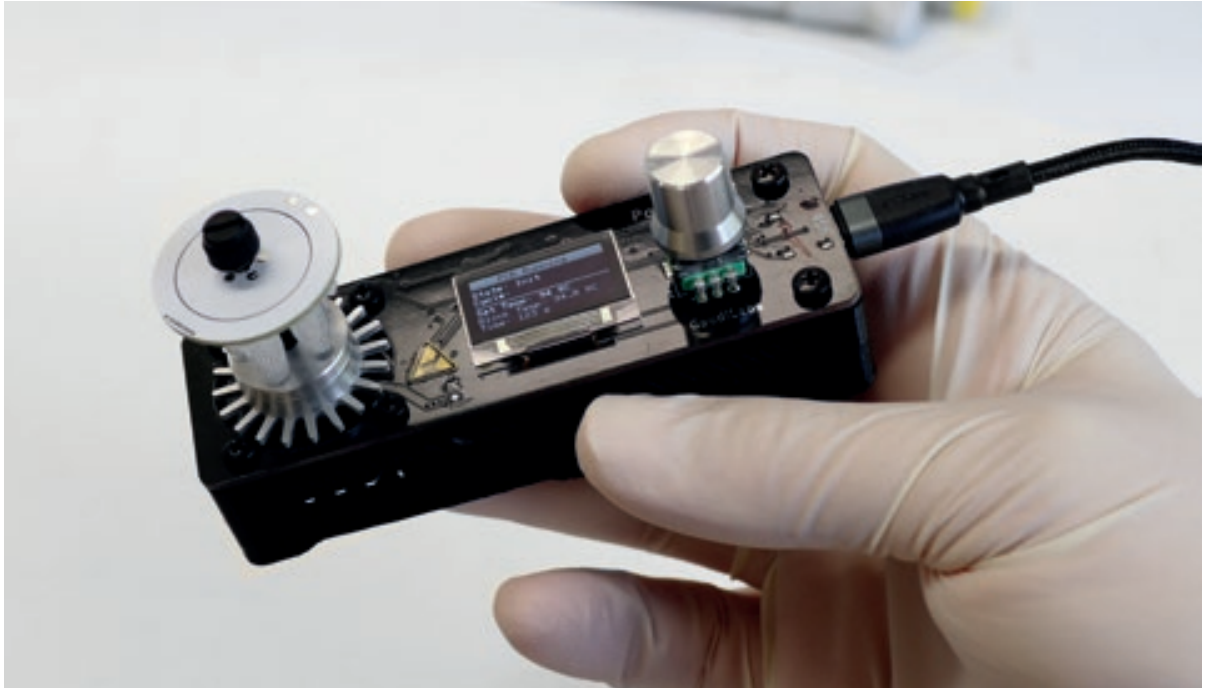

4. Plateforme miniature pour PCR (polymerase chain reaction) (www.gaudi.ch).

copies d'un ADN ») (fig. 4). Un autre exemple récent est celui de la structure collaborative parisienne Echopen dont le projet, la réalisation d'un écho-stéthoscope (sonde d'échographie connectée à un smartphone ou à une tablette) pour un cout inférieur à $1000 €$, est soutenu notamment par la fondation Pierre Fabre et l'APHP.

Des entreprises ont adopté un modèle très proche de celui des Fab Labs avec des développements en source ouverte, que ce soit pour le matériel ou pour la partie logicielle. C'est le cas de la société italienne Novaetech, qui développe les microbalances à quartz openQCM.

\section{Le cas de l'Afrique}

Des Fab Labs ont été créés ab initio sur le continent africain, comme le Woelab à Lomé (Togo) qui a construit sa propre imprimante 3D à partir de rebuts d'informatique (imprimantes, scanners, etc.). La fondation Orange et l'Agence universitaire de la francophonie (AUF) ont contribué à la naissance d'un Fab Lab à Yaoundé au Cameroun.

En ce qui concerne l'instrumentation scientifique proprement dite (principalement appuyée sur la photonique) et l'équipement pour laboratoire, le mouvement des Fab Labs a été influencé par le mouvement "Open Science Hardware", dont un atelier réunissant plusieurs acteurs s'est déroulé au CERN début mars 2016 [10]. Ce mouvement a évolué vers la structure GOSH (Global Open Source Hardware), qui a organisé plusieurs ateliers sur les différents continents. Il faut ici mentionner Josuah Pearce, professeur à l'université technologique du Michigan, qui a été le pionnier de nombreux développements en source ouverte pour le matériel [11]. Il a en particulier montré l'intérêt de l'impression 3D pour la fabrication de matériel de travaux pratiques d'optique [12] et d'équipements de laboratoire.

Nous voudrions aussi mettre en exergue une action "vertueuse » de jeunes physiciens des universités de Cambridge et de Bath : ils ont développé pour les pays à faibles ressources un microscope optique totalement imprimable en 3D, qui a fait l'objet d'une publication dans Review of Scientific Instruments, et ont réussi à obtenir des financements de plusieurs universités et institutions britanniques pour subventionner un Fab Lab en Tanzanie, le STICLab (https://sticlab.co.tz/). Ainsi, ce microscope est dorénavant fabriqué en Afrique, ce qui à notre connaissance est une première ! Son évolution a fait l'objet d'une publication [13] dans MagPi, le magazine officiel gratuit du Raspberry Pi. Ses deux principales applications sont les détections de la malaria dans les globules rouges et des bactéries dans l'eau.

Une autre action internationale est à mentionner, celle de la fondation de l'Optical Society of America (OSA), qui a conçu des dispositifs pour décontaminer les masques de protection antiparticules N95 par rayonnement UV-C, permettant une réutilisation de ceux-ci quand une pénurie de masques s'installe [14]. Des bourses ont été fournies à des groupes d'étudiants de sept pays à faibles ressources
(Bangladesh, Brésil, Éthiopie, Ghana, Kenya, Mexique et Sénégal), pour qu'ils reproduisent ces dispositifs en liaison avec les hôpitaux locaux.

\section{Avantages des Fab Labs et des approches collaboratives au temps de la pandémie de Covid-19}

La structure légère, collaborative et peu hiérarchisée des Fab Labs permet une improvisation et une créativité qui sont précieuses dans les cas où l'urgence est importante. C'est ainsi que des modèles de masques semi-rigides (les NanoHacks [3] par exemple) et de visières, l'impression de pièces détachées de respirateurs, des détournements ou l'adaptation de technologies (masques pour la plongée en apnée snorkeling - fabriqués par Decathlon, dispositifs pour l'apnée du sommeil, pour les respirateurs), des écouvillons, ont été développés, en majorité en source ouverte. Citons surtout le pousse-seringue électrique conçu et fabriqué par le hackerspace Electrolab à Nanterre [15] (fig. 5), qui a été validé sur le plan médical à l'hôpital par des essais en conditions réelles au sein d'une unité COVID (voir la photo de la page 33), et qui devrait être bientôt homologué pour une production industrielle par des entreprises en 2021.

Rappelons qu'il faut impérativement disposer d'une caution médicale pour ces développements matériels. Plusieurs structures et des individuels se sont lancés dans ces initiatives. Malheureusement la fermeture des universités, grandes écoles et autres laboratoires imposée par la pandémie a limité l'utilisation de leurs imprimantes 3D pour contribuer à l'effort général dans ce domaine, même s'il a été très significatif, comme l'a démontré, en particulier, le projet collaboratif 3D4Care, qui a permis de fabriquer et de distribuer aux hôpitaux français 17500 visières de protection de mars à mai 2020, avec l'aide de nombreux Fab Labs. Un grand avantage provient de la bonne répartition des Fab Labs en France (voir la carte de la référence [6]). La production pourrait donc s'effectuer en " circuit court ", c'est-à-dire à proximité de l'endroit où ces articles sont requis, ce qui en plus fait gagner du temps pour la distribution. Il serait important d'organiser ce mouvement dans l'éventualité d'une nouvelle pandémie. 


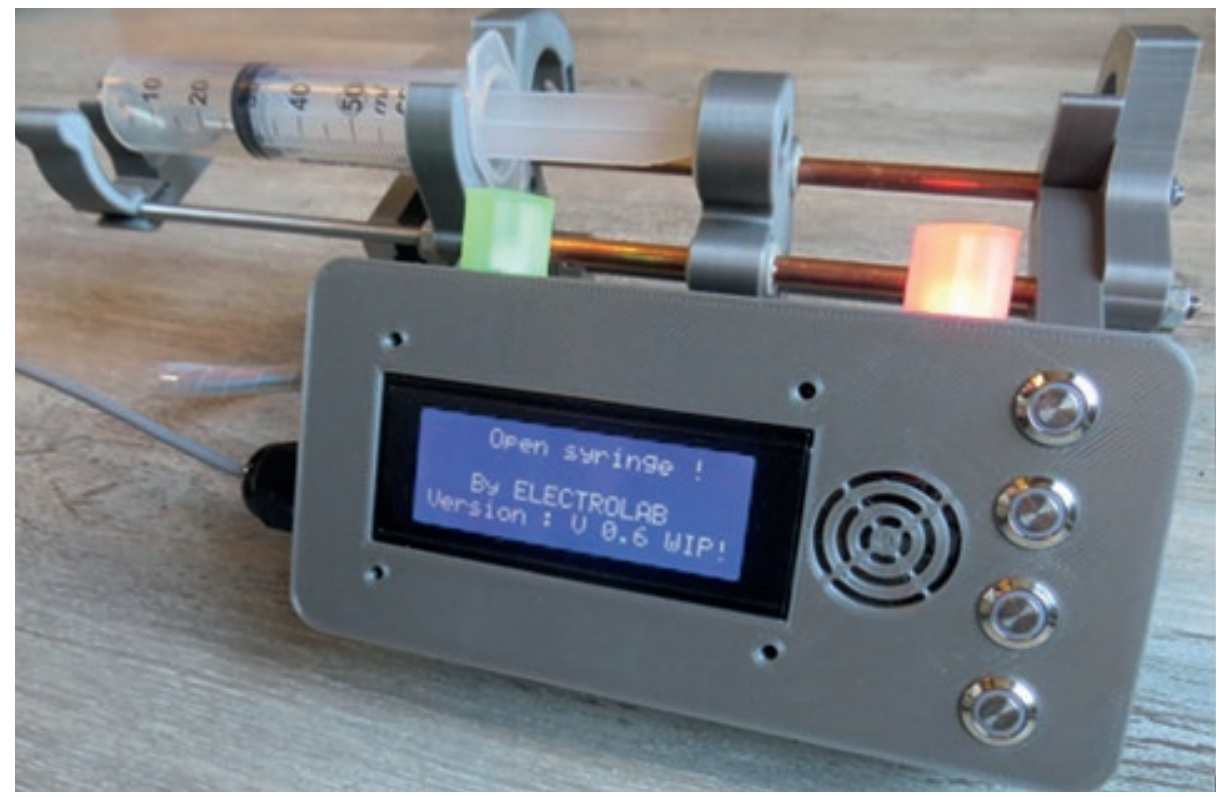

5. Le pousse-seringue électrique open source développé par Electrolab à Nanterre. Ce dispositif, créé par impression 3D et découpe laser, sera utilisé pour maintenir un débit régulier et constant de médicaments ou de liquides aux patients admis en réanimation. (Licence Creative Commons CC BY-SA 4.0).

$$
>>
$$

\section{Quel est l'avenir des Fab Labs?}

Cet avenir semble résider dans la « fabrication d'innovations " qui résultent de la grande créativité des Fab Labs, mais aussi dans la fabrication "à façon ", par exemple de pièces détachées d'appareils ménagers qui ne sont plus disponibles. Si le modèle économique est encore à inventer, on peut penser que les structures de type Fab Lab seront à l'origine de "l'artisanat numérique de demain " et que cela permettra peut-être de préserver des emplois en France [16]. Des "fermes " d'imprimantes 3D commencent à se monter, mais leurs créateurs ont du mal à dégager une marge financière suffisante leur permettant d'en vivre.

Une des techniques qui doit encore progresser est l'utilisation des poudres de métaux pour réaliser des pièces métalliques, ce qui s'effectue actuellement principalement par frittage laser, solution encore très onéreuse. De nouvelles technologies (développées par exemple par l'entreprise américaine Desktop Metal), suivies par un traitement de type MIM (moulage par injection metallique), permettent la mise en ouvre de fils contenant des particules métalliques, le matériau plastique servant de liant étant éliminé, afin d'obtenir ensuite par frittage la pièce métallique souhaitée (processus globalement long, avec un post-traitement de l'ordre de 24 heures)

Pour l'Afrique, les Fab Labs présentent une alternative pour les nombreuses universités ne disposant pas d'ateliers de mécanique, ce qui rend le prototypage très difficile.

(a) La plateforme open source Arduino est une carte électronique de faibles dimensions (quelques $\mathrm{cm}$ ), peu couteuse $(<50 €)$ et d'utilisation aisée, équipée d'un microcontrôleur et de ports d'entrée et de sortie, qui permet de programmer et commander de multiples tâches (www.arduino.cc).

(b) Le Raspberry Pi est un nano-ordinateur conçu en 2012, de la taille d'une carte de crédit, de 512 Mo de mémoire vive et de très faible prix $(\sim 25 €)$. https://fr.wikipedia.org/wiki/Raspberry $\mathrm{Pi}$

(c) La Fab Foundation recense plus de 1750 Fab Labs (au sens strict) dans le monde, dont plus de 150 en France. Dans un sens plus large, l'association Makery [6] recense en 2018 environ 380 ateliers de fabrication numérique (Fab Labs, Hackerspace, etc.) en France. Rapportés à sa population, ces chiffres placent la France, avec l'Italie, loin en tête des pays les plus investis dans cette dynamique. Remarquons qu'en Europe l'habitat, très densifié, est différent de celui de la Californie, où les populations disposent d'une importante surface potentiellement dédiée au bricolage, comme leurs grands garages.

(d) Un Hackerspace est un espace dédié au développement de logiciels open source et d'électronique, dont les plans sont disponibles et modifiables. L'accès à un Hackerspace se fait par une adhésion payante. Le détournement de technologies est une activité caractéristique de l'esprit des Hackerspaces.

\section{Références}

$1 \cdot$ www.labfab.fr/charte-fablab/

2• https://myhumankit.org

3. https://copper3d.com/hackthepandemic/

4• https://home.cern/fr/news/news/ knowledge-sharing/cern-updates-itsopen-hardware-licence

5• S. Leyronas et al., « Des communs informationnels aux communs éducationnels : les fablabs en Afrique francophone ", Projet de recherche EnCommuns (2018),

https://drive.google.com/ file/d/1vvawhllSpiU8-_SiUCoAoADzn5maG_12/view

6• www.makery.info/en/map-labs/

7• www.journals.elsevier.com/hardwarex

8- www.waterscope.org/ ; https://trendinafrica.org/openhardware

9• www.fablabs.io/labs/ictpscifablab

10• E. Gibney, "'Open-hardware' pioneers push for low-cost lab kit", Nature $\mathbf{5 3 1}$ (2016) 147-148.

11 - https://opensource.com/article/18/4/ mainstream-academia-embracesopen-source-hardware?utm campaign=intrel

12・ C. Zhang et al., "Open-Source 3D-Printable Optics Equipment", PLOS One (2013) e-59840.

13. https://magpi.raspberrypi.org/ articles/openflexure-microscope

14 M. Purschke et al., "Construction and validation of UV-C decontamination cabinets for filtering facepiece respirators", Applied Optics 59 (2020) 7585-7595. (https://doi.org/10.1364/ A0.401602)

15• www.fondationdelavenir.org/ crise-covid-19-creation-de-pousseseringues-electriques-leclairagetechnique-de-yannick-avelino-ingenieur-sein-delectrolab/

16. S. Caulier et D. Géneau, "L'industrie se fie à l'impression », Le Monde (14 mars 2016).

(e) Par exemple Instructable (www.instructables.com/), Hackteria (www.hackteria.org/),

Thingiverse (www.thingiverse.com/) exclusivement dédié à l'impression 3D, etc. 